\title{
The Theory of Contours, and its Applications in Physical Science.
}

By W. Peddre.

\section{PART I.}

1. The word contour is largely used in ordinary language, but its meaning, when so used, is in general very different from its meaning as a scientific term. We speak of the contour of a hill, a cloud, a country, and so on ; meaning usually a profile or an outline,-sometimes a particular outline only. Yet, even in this popular use of the word, we have an indication of its more exact significance. Thus, we see that the visible horizon, if we consider it to be a contour line, is the curve in which the earth's surface is met by its tangent-cone the vertex of which is at the observer's eye. The tangent-surface has a constant characteristic; and it is this possession of a distinctive property by all surfaces which give rise to contour lines, which furnishes the reason for the peculiar applicability of the method of contours to physical problems.

2. The word is used in a more special sense by ordnance surveyors. In their terminology a contour line is a line drawn on a map of a country through points which are at a constant height above mean sea-level. Here again we have a quantity which is constant all along the line, and also all over the surface which gives rise to the line by its intersection with the eurth. This is a level surface, or surface of equilibrium. It is approximately true that the special characteristic is constant height above sea-level, so long as the area considered is so small that gravity has sensibly the same value all over it. Because of the variation of gravity from point to point of the earth's surface, and because of the earth's rotation, dtc., it is not true that the mutual distance of two level surfaces is of uniform value. What is really constant is the kinetic energy acquired by a body in falling from the upper level to the lower by any path.

So, in extension of the third order, a contour may be the intersection of a surface by a surface over which some quantity is constant.

3. This idea may be extended to objects of other dimensions than the second, and extension of other order than the third. We may state generally that the contour of an object of $\mathrm{n}$ dimensions, existing 
in extension of the $(\mathrm{n}+1)^{\text {th }}$ order, is the intersection of it with an object of $\mathrm{n}$ dimensions at every point of which some quantity is constant. It is, therefore, of $(n-1)$ dimensions. But, since in extension of the $(n+1)^{\text {th }}$ order, we have objects of all positive dimensions up the $(n+1)^{\text {th }}$ inclusive; in such extension, we may have contours of all positive dimensions up to the $n^{\text {th }}$ inclusive. The contours of a curve are points; of a surface, curves; of a solid, surfaces; of a fourdimensional object, solids; and so on. Hence, in ordinary extension we may have point, curve, and surface, contours.

4. Since space is of three dimensions, a point in space may have three degrees of translational freedom,-it may be free to move in any three mutually perpendicular directions successively or simultaneously. We are said to introduce a degree of constraint when we take away one of these degrees of freedom. If we prevent the exercise of one kind of freedom, unless a second is simultaneously exercised, we introduce a degree of constraint in the most general way possible. 'The moving point is then forced to remain on some definite surface, and the nature of the constraint is best shown by writing down the equation to the surface, the most general being of the form $f(x, y, x)=0$. If another such constraint be imposed, the moving point must remain in positions common to both the corresponding surfaces. Hence, it must move in a definite curve. Three such equations determine the position of a point. They may degenerate into $f_{1}(x)=0, f_{1}(y)=0, f_{1}(z)=0$, where $f_{1}(x)$ is some function of $x$ of the first degree, \&c. ; that is to say, the position of the point is determined by the intersection of three planes parallel to the co-ordinate planes.

5. A curve being of one dimension, its contours are points,-the Foints of intersection of it with curves along each of which some quantity is constant, although it differs in magnitude from one curve to another. These points may be projected upon any line in the plane $(x, y)$.

We shall consider first a plane curve, and let its plane be taken as that of $(x, y)$. We have the equations $f_{n}(x, y)=0$ and $f_{1}(z)=0$, where the suffix denotes the degree of the equation. Instead of the second equation, we may put $z=0$, as this simply makes the plane of the curve that of $(x, y)$. The equations to the curves, along which some quantity, say $c$, is constant, may be written in the general form $\phi_{w}(x, y, c)=0$. In the different curves of the system, $c$ has different values. As a particular example, the curves unight be 
circles of different radii. Again, the equation might be $\phi_{1}(y, c)=0$. This represents a series of lines parallel to the axis of $x$. It furnishes the simplest case for consideration, and, at the same time, the most useful.

From the reference already made $(\S 2)$ to contour maps, it is evident that the mutual variation of three quantities can be represented upon a plane surface by means of lines. So, in the case considered, the mutual variation of two quantities can be represented upon a right line. The curve in fig. 20 is intersected by lines parallel to the axis of $x$, and the points of intersection are projected up $)$ n the axis, and designated by numbers giving the varions values of $y$ corresponding to the values of $x$. If the curve be continuous a maximum or minimum value of $y$ exists between two equal values. It is a maximum if, as $x$ increases from its least value corresponding to the given value of $y, y$ first increases and then diminishes. It is a minimum if $y$ first diminishes and then increases. The steepness of slope is shown by the closeness of the contours for equal increments of $y$, and its direction is shown by the order in which the values of $y$ occur as regards numerical magnitude when $x$ increases. Similarly, the existence of a maximum-minimum value is indicated, and so on.

The contours of a closed oval and of a tigure-of-eight curve, are shown in figs. 21 and 23.

6. Hitherto we have considered only plane curves. In the case of tortuous curves, it will be most convenient to obtain the contours by cutting the curve by surfaces over which some quantity is constant. As a particular case, these surfaces may be planes perpendicular to the z-axis, the equations to which may be written in the general form $f_{1}(z, c)=0$. Obviously, the position of a moving point in space is representable by a tortuous curve. Its position at any time can be got from the curve, if the value of the time in terms any one of the co-ordinates, say $z$ is known, for we should then only have to cut the curve by the plane ${ }_{1} f_{\mathrm{n}}(z, t)=0$, where $t$ represents the time, and $f_{\mathrm{a}}$ is a functional symbol showing that the equation is of the first degree in $z$ but may be of any degree in $t$. This condition is rendered necessary by the fact that the point must be in one definite position at a given time, but may occupy the same position at different times. If we consider a number of such curves traced out in space by moving material points, we can, by cutting the curves by planes corresponding to a definite time and projecting the points so got upon any co-ordinate plane, obtain the diagram of configuration 
of the material system at that time. It is evident that, in general, the plane corresponding to a definite time will be different for each curve. The disadvantage so entailed may be got rid of by using trilinear co-ordinates to indicate the position of the point when the time is giren. If the curve be cut by any plane, the distances of the point of intersection from three intersecting lines in that plane give the $x, y$, and $z$ co-ordinates at an instant. This instant may be determined by the distance of the plane from a given fixed plane parallel to it. The curves, which are now taken to represent the positions of the points, are not the actual curves traced out by the points in their motion through space. But the diagram of configuration obtained from them has the advantage of showing at once the relative values of all the co-ordinates of all the points, whereas, in the Cartesian system this could not be done without projecting on all three co-ordinate planes. The triangles of reference will be similar whatever be the plane by which the curves are cut, but they will not necessarily be of the same magnitude. The similarity is necessary in order to give the proper ratios of the co-ordinates, the different magnitude is necessary in order to give the proper values of the co-ordinates. Fig. 22 shows the contours of curves $a, b$, by planes corresponding to times $t_{1}, t_{\mathbf{2}}$.

By the aid of this diagram, the diagram of total displacements in a given time may be constructed. And by taking the displacements in $\frac{1}{n}$ th of the unit of time and magnifying them $n$ times, the diayram of velocities can be got. Similarly, the diagrams of acceleration, furces, and so on, may be represented as the contours of curves. The curves, from which the diagrams of velocities, \&c., are obtained, are, of course, different from the original curves representing the position of the moving points. In the case of velocities, for example, they are the hodographs of the original curves on this trilinear system of reference. 